\title{
Evropiyum içeren lüminesans özelliği gösteren metal- organik malzemelerin üretimi ve yapılarının araştırılması
}

\author{
Adem DÖNMEZ ${ }^{1,2, *}$, Uğur ERKARSLAN ${ }^{1}$, Şehmus KARADENİ' ${ }^{1}$ \\ ${ }^{1}$ Muğla Sitkı Koçman Üniversitesi, Fen Fakültesi, Fizik Bölümü, Muğla. \\ ${ }^{2}$ Muğla Sitkı Koçman Üniversitesi, Bilimsel Araştırma Projeleri Koordinasyon Birimi, Muğla. \\ Gelis Tarihi (Recived Date): 18.08 .2017 \\ Kabul Tarihi (Accepted Date): 14.11.2017
}

Özet

Molekül yapılarında birleştirici olarak ligand ve bağlayıcı olarak metal merkezler bulunan, uzaysal mimarisi özgün olan Metal organik çerçevelerin (MOÇ), moleküller arası uzaklıkları büyük olduğundan geniş hacimli boşluklara sahip olma özelliği ve yüzey alanlarının geniş olması nedeniyle manyetizma, lüminesans, hidrojen depolama, gaz absorbsiyonu, optik sensör gibi bir çok fiziksel/kimyasal/elektro-optik uygulama alanları mevcuttur.

Bu çalışmada; Lantanit Metal Organik Çerçeveler içeren; [Eu(2-STP)], [2-STP=2-sülfoterefitalat] (1) hidrotermal yöntem ile sentezlenmiştir. Sentezlenen bu malzemenin yapısal analizi; Toz kristal X-ışını kırınımı, katı hal UV ve FT-IR spektroskopisi teknikleri kullanılarak açığa çıkartılmıştır. Malzemenin katı hal fotolüminesans özellikleri oda sıcaklığında görünür bölge ve yakın kızıl ötesi bölgede ölçülmüşse "anten" görevi yapan 2-STP ligandın dan Eu III iyonuna enerji transfer mekanizması detayll olarak tartışılmıştır.

Anahtar Kelimeler: MOÇ, lüminesans, Evropiyum.

\section{Synthesis and structural analysis of europium metal-organic materials which show luminescence properties}

\begin{abstract}
Metal organic frameworks (MOFs), which is unique in spatial architecture and has ligands and metal centers as linkers and as a binding in molecular structures, due to their large intermolecular distances and their large surface area, they have many physical/chemical/electro-optic application areas such as magnetism, luminescence, hydrogen storage, gas absorption, optical sensor and so on.

In this work; Lanthanide Metal Organic Frameworks; [Eu(2-STP)], (2-STP = 2-sulfoterephthalate) $(1)$ were synthesized using hydrothermal method. The structural analysis of synthesized material has been characterized by powder X-ray diffraction, solid state UV and FT-IR spectra. The solid state photoluminescence properties of the material were measured at room temperature in visible and NIR region, and the energy transfer mechanism from 2-STP ligand to the central Eu III ion via an "antenna effect" was discussed in details.
\end{abstract}

Keywords: MOF, luminescence, Europium.

\footnotetext{
* Adem DÖNMEZ, adonmez@mu.edu.tr, http://orcid.org/0000-0002-9773-0493

Uğur ERKARSLAN, egur@mu.edu.tr, http://orcid.org/0000-0001-7667-2151

Şehmuz KARADENIZ, sehmuskaradenizz@gmail.com, http://orcid.org/0000-0002-7065-4507
} 


\section{Giriş}

Metal-Organik çerçeveler (MOÇ) üzerinde son 20 yıldır çok çalışılan, endüstride uygulama alanları olan gözenekli malzemelerdir [1-6]. Lüminesans özellik gösteren MOÇ ler, uygulama potansiyelleri nedeniyle araştırmacılar tarafından ilgiyle üzerinde durulan konulardan biridir. Çoban ve arkadaşları Ho(III) iyonu içeren bir kompleks de, ligand dan Ho(III) iyonuna yük transferi ile fotolüminesans özelliği rapor etmişlerdir [7]. Yapılan başka bir çalışmalarında ise $\mathrm{Ho}(\mathrm{III})$ ve $\mathrm{Sm}(\mathrm{III})$ içeren komplekslerinde, ligandların "Anten Etkisinin" olduğunu ve Ln(III) iyonlarına enerji transferiyle şiddetli fotolüminesans özelliği gözlemlemişlerdir [8]. Farklı ligandlar kullanılarak elde edilen Eu(III) içeren malzemeler, keskin emisyon bantları, yüksek duyarlık ve uzun dalga boylu ışıma yapması ve f-f geçişlerinde uzun lüminesans ömrüne sahip olması nedeni ile son zamanlarda yoğun şekilde çalışılmıştır [9-14]. Ancak Eu(III) de diğer lantanitler gibi parite seçim kuralı sonucunda yasaklı f-f geçişi nedeniyle düşük soğurma spektrumuna sahiptir. Bu iyonlardan şiddetli lüminesans elde edebilmenin yolu, iyi birer 1 şı soğurucu olan organik ligandların kullanılmasıdır. Organik ligandlar UV bölgesinde soğurdukları enerjiyi lantanit iyonun $4 f$ rezonans seviyelerine uyarma enerjisi olarak aktarırlar. $\mathrm{Bu}$ olaya literatürde 'Anten' etkisi denmektedir. Lantanit kompleksleri bu özelliklerinden dolayı ligandlarla sentezlendiği zaman daha şiddetli lüminesans özellik gösterebilirler [7].

Bu çalışmada; 2-STP ligandı ile Eu(III) iyonu içeren kompleks (1) hidrotermal yöntem kullanılarak toz kristal olarak elde edilmiş ve literatürde daha önceden rapor edilmiş tek kristal yapısı ile karşılaştırılmıştır [15]. Kompleks (1) in katı hal UV ve IR ölçümleri, görünür ve yakın kızılötesi bölgede ayrıntılı fotolüminesans analizleri ilk kez bu çalışmada yapılmıştır. 2-STP ligandının "Anten Etkisi” gösterdiği ve Eu(III) iyonun yaptığ1 lüminesansı şiddetlendirdiği gözlenmiştir ve bu olay enerji transfer mekanizmaları ile ayrıntılı olarak açıklanmıştır.

\section{Materyal ve metot}

2-sülfoterefitalik asit ve $\mathrm{Eu}\left(\mathrm{CI}_{3}\right)_{3}$ x $5 . \mathrm{H}_{2} \mathrm{O}$, ticari firmalardan satın alınarak, herhangi bir kimyasal saflaştırma işlemine tabii tutulmadan kullanılmıştır. Malzemenin yapisal analizi; Philips X'Pert Pro marka toz kırınım cihazı, Perkin-Elmer Spectrum 65 FT-IR spektrometresi ve Ocean Optics Maya 2000 Pro marka katı UV spektrometre ölçümleri ile yapılmıştır. Fotolüminesans ölçüm verileri ise Spectra Physics model Nd-YLF $349 \mathrm{~nm}$ dalga boylu lazer kaynağı kullanılarak, Perkin Elmer LS 55 moleküler florometre ve Andor Solis SR 500i-BL model spektrometre ile oda sicaklığında elde edilmiştir.

\subsection{Malzemenin sentezlenmesi}

1 mmol (0.269 g) 2-sülfoterefitalik asit oda sıcaklığında $10 \mathrm{~mL}$ saf suda çözülmüştür. Daha sonra çözelti manyetik karıştırıcıda karışırken üzerine çözünen $1 \mathrm{mmol}(0.366 \mathrm{~g}) \mathrm{Eu}\left(\mathrm{Cl}_{3}\right)_{3}$ x $5 \mathrm{H}_{2} \mathrm{O}$ ekleyip 20 dakika manyetik karıştırıcı ile karıștırılmıştır. Hazırlanan çözeltinin oda Sıcaklığında ilk Ph değeri 2.57 olarak gözlenmiştir. Çözeltiye damla damla $\mathrm{NaOH}$ eklemek suretiyle $\mathrm{Ph}$ değeri 4.01 değerine yükseltilerek, çözelti tekrar manyetik karıştırıcıda kaynatılarak karıştırılmıştır. Elde edilen karışım, yüksek basınca dayanıklı reaktörlerde oda sicaklığından $120{ }^{\circ} \mathrm{C}$ dereceye 1sıtılıp, 50 saat bu sicaklıkta tutulmuş ve 24 saatte oda sıcaklığına düşürülecek şekilde programlanmış etüvde malzeme toz kristal olarak elde edilmiştir. Elde edilen toz kristaller süzme kâğıdıyla ayrıştırılmıştır. Kompleks (1) 'in şematik gösterimi Şekil 2.1' de verilmiştir.<smiles>CCOS(=O)(=O)c1cc(C(=O)O)ccc1C(=O)O</smiles>

Şekil 2.1: Kompleks (1)'in şematik gösterimi.

\section{Araştırma bulguları}

\subsection{Kompleks (1)’in toz kristal ölçümüi}

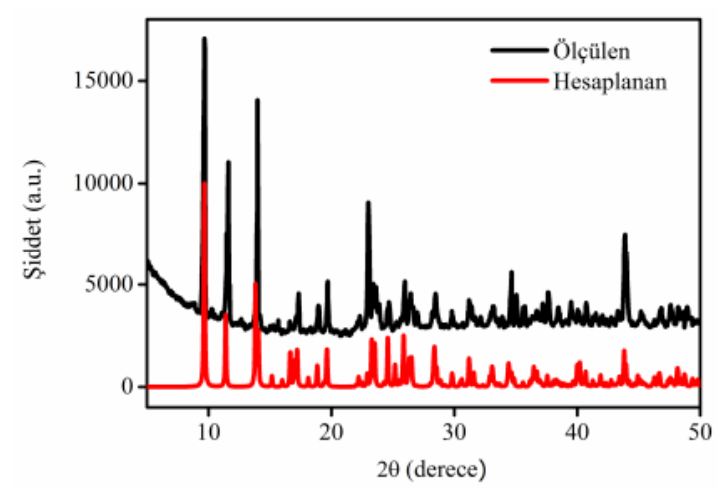

Şekil 3.1: Kompleks (1) için X-ışını toz kırınımı desenleri (Kırmız1-CIF den simülasyon ile hesaplanan, Siyah-Deneysel olarak ölçülen data)

Kompleks (1)' in X-ışını toz kırınımı şiddet verilerinden elde edilen veriler, Mercury bilgisayar programı kullanılarak literatürdeki tek kristal yapısı ile karşılaştırılmıştır [15]. Toz kristal ölçümünden 
elde edilen grafik, "ölçülen” olarak, tek kristal verileri kullanılarak Mercury bilgisayar programı ile elde edilen grafik ise "hesaplanan" olarak isimlendirilmiş ve grafikler karşılaştırmalı olarak aşağıda verilmiştir (Şekil 3.1). Grafikler incelendiğinde; kompleks (1) de ölçülen ve hesaplanan piklerin uyumlu olduğu görülmüştür. Gözlenen piklerin uyumlu olması incelenen kompleksin saf olduğunu göstermektedir.

\subsection{Kompleks (1)'in IR spektrumu}

Kompleks (1) in ve serbest ligand 1 2-STP' nin IR spektrumları ayrı ayrı alınmış (Şekil 3.2) ve Tablo 3.1'de karş1laştırılmıştır. 3591-3505 $\mathrm{cm}^{-1}$ de gözlenen pikler koordine su molekülünden gelen $v(\mathrm{O}-\mathrm{H})$ gerilme titreşimlerinden kaynaklandığ düşünülmektedir [16]. 2988-1691 $\mathrm{cm}^{-1}$ bölgesinde meydana gelen güçlü titreşim piklerinin görülmemesi $\mathrm{SO}_{3} \mathrm{H}$ gruplarının protondan arındırıldığını göstermektedir. 1561-1392 $\mathrm{cm}^{-1} \mathrm{de}$ gözlenen şiddetli pikler, $v_{\mathrm{as}}(\mathrm{COO})$ ve $v_{\mathrm{s}}(\mathrm{COO})$ karboksilat gruplarının titreşimlerinden kaynaklanmaktadır. Kompleks (1) de $v_{\text {as }}$ ve $v_{\mathrm{s}}$ titreşim pikleri arasında meydana gelen $169 \mathrm{~cm}^{-1}$ lik fark, karboksilat gruplarının şelat modunda metal iyonu ile koordine olduğunu göstermektedir [17]. Ayrıca $\mathrm{SO}_{3}$ gruplarının karakteristik titreşimleri $1309-1030 \mathrm{~cm}^{-1}$ bölgesinde görülmektedir [18].

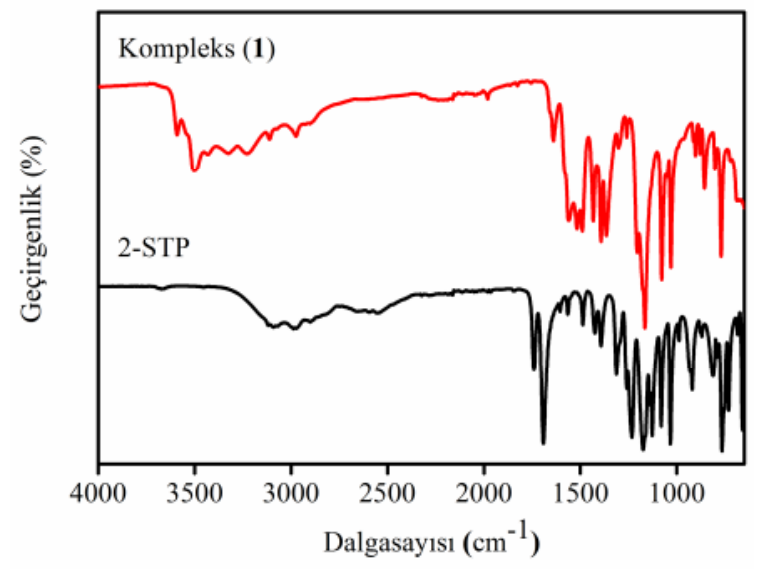

Şekil 3.2: 2-STP ligandı ile Kompleks (1)'in IR spektrumları

Tablo 3.1: 2-STP ve Kompleks (1)'e ait IR ölçüm verileri.

\begin{tabular}{ccccccc}
\hline Malzeme & $v(\mathrm{O}-\mathrm{H})$ & $v(\mathrm{COOH}, \mathrm{SO} 3 \mathrm{H})$ & $v a s(\mathrm{COO})$ & $v \mathrm{~s}(\mathrm{COO})$ & $v(\mathrm{SO} 3)$ & $v \operatorname{vas}(\mathrm{COO})-\mathrm{vs}(\mathrm{COO})$ \\
\hline 2-STP & & $2988-1691$ & 1565 & 1392 & $1312-1032$ & \\
Kompleks $(\mathbf{1})$ & $3591-$ & - & 1561 & 1392 & $1309-1030$ & 169 \\
\hline
\end{tabular}

\subsection{Kompleks (1)'in UV spektrumu}

Kompleks (1)' in ve serbest ligand1 2-STP nin kat1 halde UV spektrumları 240-550 nm de ayrı ayrı alınmış ve karşılaştırmalı grafikleri Şekil 3.3' de verilmiştir. 2-STP ligandı için maksimumu $375 \mathrm{~nm}$ dalgaboyu değerinde bir soğurma bandı gözlenirken bu bant kompleks (1)'de $483 \mathrm{~nm}$ ye kaymıştır. Gözlenen soğurma bantları muhtemelen serbest ligandlardaki $\pi-\pi^{*}$ veya $\mathrm{n}-\pi^{*}$ geçişlerinden kaynaklanmaktadır [19]. Kompleks (1)'in soğurma bantı 2-STP ligandın soğurma bantı ile kıyaslandığında daha yüksek dalga boyuna doğru kaymaktadır. Bu kaymanın sebebi, ligandın oksijen atomları ile $\mathrm{Eu}(\mathrm{III})$ iyonlarının koordinasyon yapmasidir [20].

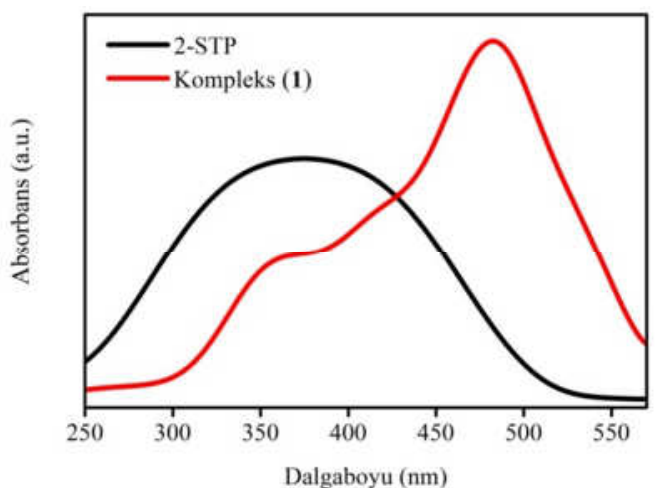

Şekil 3.3: 2-STP ligandı ile Kompleks (1)' in UV spektrumları

\subsection{Kompleks (1)'in fotolüminesans özelliklerinin incelenmesi}

Kompleks (1) ve 2-STP ligandının fotolüminesans ölçümleri toz fazda ve oda sicaklığında $\lambda=349 \mathrm{~nm}$ lazer uyarması ile yapılmıştır. 2-STP ligandının emisyon spektrumu Şekil 3.4'de, kompleks (1) için görünür bölge emisyon spektrumu Şekil 3.5 de ve NIR bölge emisyon spektrumu ise Şekil 3.6 da verilmektedir.

2-STP ligandının emisyon spektrumu incelendiğinde, geniş bant spektrumunun maksimum olduğu dalga boyları 477, 511 ve 558 nm'de yeşil emisyon bandı göstermektedir. Bu maksimum dalga boylarına karşılık gelen geçişler $n \rightarrow \pi^{*}$ veya $\pi \rightarrow \pi^{*}$ elektronik geçişlerinden kaynaklanmaktadır [19].

Kompleks (1) şiddetli kırmızı 1şıma yapmaktadır. Kompleks (1) de Eu(III) iyonunun görünür bölgede beş karakteristik ${ }^{5} \mathrm{D}_{0} \rightarrow{ }^{7} \mathrm{~F}_{\mathrm{J}} \quad(\mathrm{J}:$ : 0-4) emisyon piki gözlenmiştir. $\mathrm{Bu}$ pikler sırası ile $579 \mathrm{~nm}$ de ${ }^{5} \mathrm{D}_{0} \rightarrow{ }^{7} \mathrm{~F}_{0}, 593 \mathrm{~nm}$ de ${ }^{5} \mathrm{D}_{0} \rightarrow{ }^{7} \mathrm{~F}_{1}, 616 \mathrm{~nm}$ de ${ }^{5} \mathrm{D}_{0} \rightarrow{ }^{7} \mathrm{~F}_{2}$, $652 \mathrm{~nm}$ de ${ }^{5} \mathrm{D}_{0} \rightarrow{ }^{7} \mathrm{~F}_{3}, 698 \mathrm{~nm}$ de ise ${ }^{5} \mathrm{D}_{0} \rightarrow{ }^{7} \mathrm{~F}_{4}$ geçişlerine karşılık gelmektedir. 


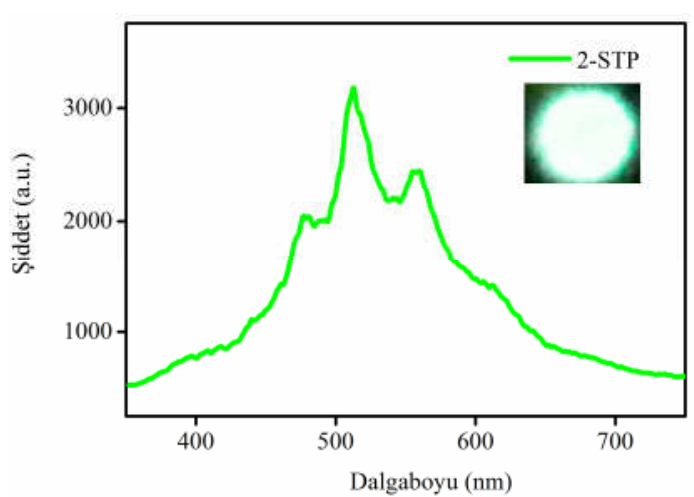

Şekil 3.4: 2-STP ligandına ait emisyon spektrumu

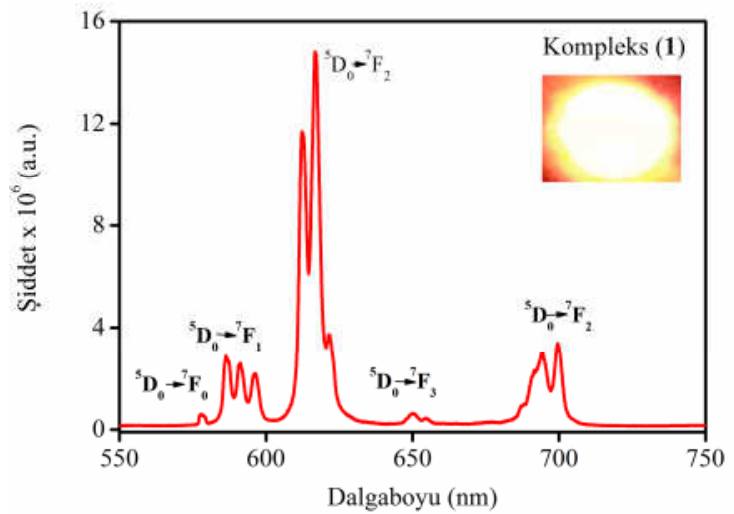

Şekil 3.5: Kompleks (1)'in görünür bölge emisyon spektrumu

Kompleks (1) NIR bölgede ise 3 karakteristik emisyon piki vermektedir (Şekil 3.6). NIR bölgedeki emisyon pikleri görünür bölgedekilere göre daha düşük şiddetlidir. Gözlenen emisyon piklerine uygun elektronik geçişleri; ${ }^{7} \mathrm{~F}_{6} \rightarrow{ }^{7} \mathrm{~F}_{0}(1160 \mathrm{~nm}),{ }^{7} \mathrm{~F}_{5} \rightarrow{ }^{7} \mathrm{~F}_{0}$ $(1210 \mathrm{~nm}) \quad$ ve ${ }^{7} \mathrm{~F}_{4} \rightarrow{ }^{7} \mathrm{~F}_{0} \quad(1370 \mathrm{~nm}) \quad$ olarak belirlenmiştir.

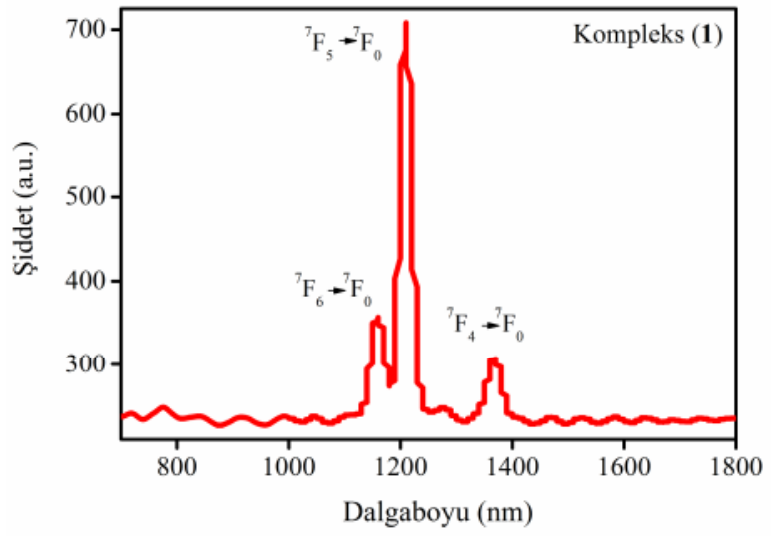

Şekil 3.6: Kompleks (1)' in NIR bölge emisyon spektrumu

Ln(III) iyonlarının direk uyarılması, lantanit iyonlarının düşük soğurma yaratan parite (Laporte) seçme kuralları ile yasaklanmış $\mathrm{f}$ - $\mathrm{f}$ geçiş sebebiyle nerdeyse imkansızdır. $\mathrm{Bu}$ nedenle, araştırmacılar 1şığ $\mathrm{UV}$ bandında soğuran ve anten etkisiyle (Şekil
3.7) uyarılma enerjisini ligandlardan lantanit iyonlarına çeviren, lantanit iyonları için uygun kromoforlara sahip organik ligandların kullanımını önermişleridir. Anten etkisi komplekslerin uyarılma spektrumu ve ligandların soğurma spektrumlarının örtüşmesinden görülmektedir.

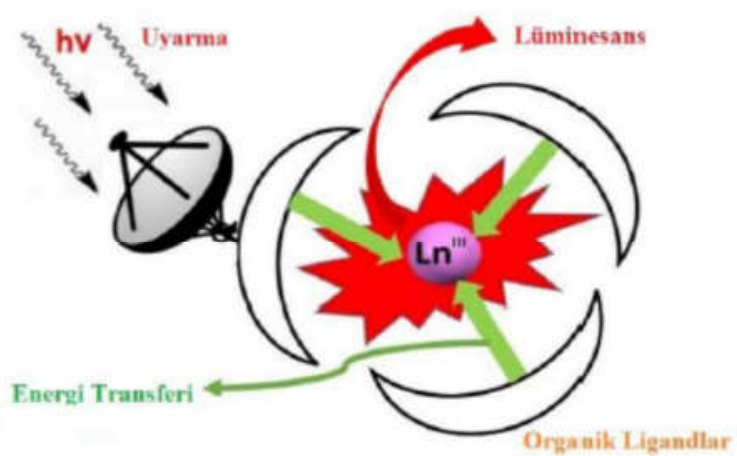

Şekil 3.7: Anten etkisi ile Eu(III) iyonlarının lüminesans özelliği kazanmalarının şematik gösterimi [7]

Kompleks (1)' in uyarılma ve 2-STP ligandının soğurma spektrumları Şekil 3.8' de gösterilmiştir. Kompleks (1)' in uyarma spektrumunun 2-STP ligandının soğurma spektrumu ile çok iyi şekilde örtüşmesi, liganddan $\mathrm{Eu}(\mathrm{III})$ iyonuna enerji transferinin gerçekleştiğini ve 2-STP ligandının "Anten etkisi” ni göstermektedir.

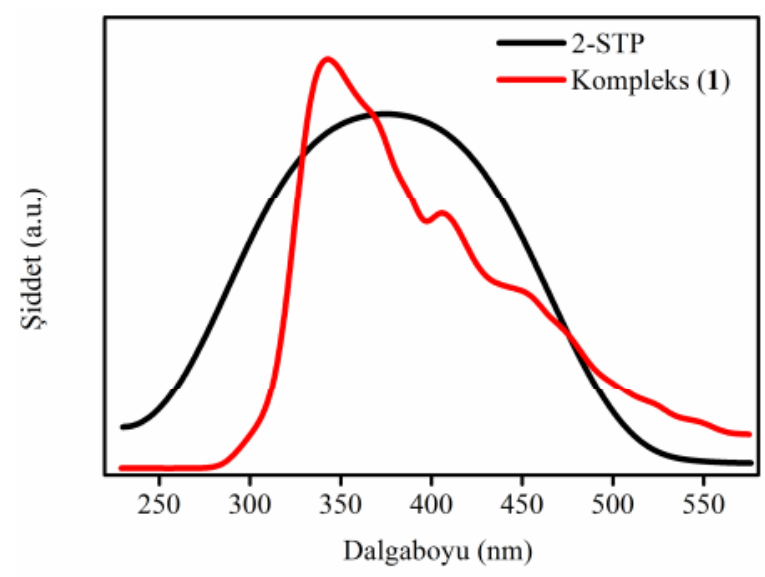

Șekil 3.8: Kompleks (1)'in uyarılma ve 2-STP ligandının soğurma spektrumları

\section{Tartışma ve sonuç}

Bu çalışmada; 2-sülfoterefitalik asit (2-STP) ligandı ve $\mathrm{Eu}\left(\mathrm{Cl}_{3}\right)_{3} \times 5 . \mathrm{H}_{2} \mathrm{O}$ tuzu kullanılarak yeni $\mathrm{Eu}$ (III) tabanlı [Eu(2-STP)] (1) kompleksi hidrotermal koşullar altında başarıyla sentezlendi. Kompleks 1 in yapısal analizi toz kristal XRD ölçümü, katı hal UVVis ve FTIR ölçümleri ile yapılmıştır. Toz kristal XRD ölçümü sonucunda gözlenen piklerin hesaplanan ile uyumlu olması incelenen kompleks 1 in saf olduğunu göstermektedir. Buna ek olarak 
kompleks 1 in ve serbest ligandının UV ve IR ölçüm sonuçları incelendiğinde kristal yapı içindeki bağlanmanın tek kristal x-1şınları analizinden elde edilen sonuçlar ile uyumlu olduğu gözlenmiştir. Ayrıca kompleks 1 in oda sıcaklığında görünür ve NIR bölgede katı hal fotolüminesans ölçümleri yapılmıștır. 2-STP serbest ligandı ile kompleks (1) in fotolüminesans ölçümleri karşılaştırıldığında; serbest ligand 477, 511 ve 558 nm' de yeşil emisyon verirken, kompleks (1) $\mathrm{Eu}(\mathrm{III})$ iyonunun görünür bölgede beş karakteristik emisyon piki ve NIR bölgede 3 karakteristik emisyon piki vermekte ve şiddetli kırmızı ışıma yapmaktadır. Bu pikler sırası ile $579 \mathrm{~nm}$ de ${ }^{5} \mathrm{D}_{0} \rightarrow{ }^{7} \mathrm{~F}_{0}, 593 \mathrm{~nm}$ de ${ }^{5} \mathrm{D}_{0} \rightarrow{ }^{7} \mathrm{~F}_{1}, 616$ $\mathrm{nm}$ de ${ }^{5} \mathrm{D}_{0} \rightarrow{ }^{7} \mathrm{~F}_{2}, 652 \mathrm{~nm}$ de ${ }^{5} \mathrm{D}_{0} \rightarrow{ }^{7} \mathrm{~F}_{3}, 698 \mathrm{~nm}$ de ${ }^{5} \mathrm{D}_{0} \rightarrow{ }^{7} \mathrm{~F}_{4}, 1160 \mathrm{~nm} \mathrm{de}{ }^{7} \mathrm{~F}_{6} \rightarrow{ }^{7} \mathrm{~F}_{0}, 1210 \mathrm{~nm} \mathrm{de}{ }^{7} \mathrm{~F}_{5} \rightarrow{ }^{7} \mathrm{~F}_{0}$ ve $1370 \mathrm{~nm}$ de ${ }^{7} \mathrm{~F}_{4} \rightarrow{ }^{7} \mathrm{~F}_{0}$ geçişlerine karşıllk gelmektedir. Kompleks (1) in görünür ve NIR bölge spektrumları incelendiğinde ligand kaynaklı herhangi bir geniş bant emisyonunun tespit

\section{Kaynaklar}

[1] Zhao, B., Chen, X.Y., Cheng, P., Liao, D.Z., Yan, S.P. ve Jiang, Z.H., Coordination polymers containing $1 \mathrm{D}$ channels as selective luminescent probes, Journal of Amarican Chemical Society, 126, 15394-15395, (2004).

[2] Guo, Z.Y., Wu, H., Srinivas, G., Zhou, Y.M., Xiang, S.C., Chen, Z.X., Yang, Y. T., Zhou, W., O'Keeffe, M. ve Chen, B.L., A metalorganic framework with optimized open metal sites and pore spaces for high methane storage at room temperature, Angewandte Chemie International Edition, 50, 31783181, (2011).

[3] Jiang, C.Y., Wu, T., Zheng, S.T., Zhao, X., Lin, Q.P., Bu, X.H. ve Feng, P.Y., A nineconnected mixed-ligand nickel-organic framework and its gas absorption properties, Crystal Growth \& Design, 11, 3713-3716, (2011).

[4] Getman, R.B., Bae, Y.S., Wilmer, C.E. ve Snurr, R.Q., Review and analysis of molecular simulations of methane, hydrogen, and acetylene storage in metal-organic frameworks, Chemical Reviews, 112, 703723, (2012).

[5] Bao, S.S., Ma, L.F., Wang, Y., Fang, L., Zhu, C.J., Li, Y.Z. ve Zheng, L.M., Aniondirected self-assembly of lanthanide-notp compounds and their fluorescence, magnetic, and catalytic properties, Chemistry - A European Journal, 13, 2333-2343, (2007).

[6] Lin, Z.J., Yang, Z., Liu, T.F., Huang, Y.B. ve Cao, R., Microwave-assisted synthesis of a series of lanthanide metal-organic frameworks and gas absorption properties. edilememesi 2-STP ligandı tarafından soğurulan enerjinin Eu(III) iyonuna verimli şekilde aktarıldığ anlamına gelir. Buna ilaveten kompleks (1)' in uyarma spektrumunun 2-STP ligandının soğurma spektrumu ile iyi şekilde örtüşmesi, liganddan Eu(III) iyonuna enerji transferinin gerçekleştiğini ve 2-STP ligandının "Anten etkisi” ni göstermektedir. $\mathrm{Bu}$ umut verici fotolüminesans davranış, incelenen kompleksin 1şık yayan diyotlar (OLEDs) için olası uygulamalarına kapı açmaktadır.

\section{Teşekkür}

Bu çalışma Muğla Sıtkı Koçman Üniversitesi Bilimsel Araştırma Koordinasyon (MSKÜ - BAP) Birimi tarafından (16/042) kod numaralı proje ile desteklenmiştir. Yazarlar bu desteğinden ötürü MSKÜ BAP Birimine, ayrıca fotolüminesans ölçümleri için Balıkesir Üniversitesi Bilim ve Teknoloji Uygulama ve Araştırma Merkezine ve Dr. Mustafa Burak ÇOBAN'a teşekkür eder.

[7] Çoban, M.B., Erkarslan, U., Oylumluoglu, G., Aygün, M. ve Kara, H., Hydrothermal synthesis, crystal structure and photoluminescent properties; 3D Holmium(III) coordination polymer, Inorganica Chimica Acta, 447, 87-91, (2016).

[8] Çoban, M.B., Amjad, A., Aygun, M. ve Kara, H., Sensitization of HoIII and SmIII luminescence by efficient energy transfer from antenna ligands: Magnetic, visible and NIR photoluminescence properties of GdIII, HoIII and SmIII coordination polymers, Inorganica Chimica Acta, 455, 25-33, (2017).

[9] De Sa', G.F., Malta, O.L., de Mello D.C., Simas, A.M., Longo, R.L., Santa-Cruz P.A. ve de Silv, E.F., Spectroscopic properties and design of highly luminescent lanthanide coordination complexes, Coordination Chemistry Reviews, 196, 165-195, (2000).

[10] Wang, Z., Streobele, M., Zhang, K.L., Meyer H.J., You, X.Z. ve Yu, Z., A new family of two-dimensional lanthanide(III) coordination polymers: synthesis, structures and properties of $[\mathrm{Ln}(\mathrm{SIP})(\mathrm{H} 2 \mathrm{O}) 4] \mathrm{n}(\mathrm{Ln}=\mathrm{Eu}, \mathrm{Gd}, \mathrm{Ce}$, and $\mathrm{NaH} 2 \mathrm{SIP}=5$-sulfoisophthalic acid monosodium salt), Inorganic Chemistry Communications, 5, 230-234, (2002).

[11] Hua-Bin, Z., Chong-Bin, T., Shu-Ting, W., Jian-Di L., Zhi-Hua, L. ve Shao-Wu, D., Synthesis, structures and physical properties of new 3D lanthanide coordination polymers constructed from 1,2,4,5benzenetetracarboxylic acid, Journal of Molecular Structure, 985, 355-360, (2011). 
[12] Li-Juan, H., Ya-Jie, K., Ning, S. ve XingLing, J., A new europium fluorous metalorganic framework with pentafluorobenzoate and 1,10-phenanthroline ligands: Synthesis, structure and luminescent properties, Journal of Flourino Chemistry, 166, 122-126, (2014).

[13] Hui, B., Zhenting, W. ve Ming, H., Lanthanide metal-organic frameworks with 2,2-bipyridinepolycarboxylic acid: Synthesis, crystal structures and fluorescent properties, Inorganica Chimica Acta, 427, 112-117, (2015).

[14] Yi-Xia, R., Miao, A., Hong-Mei, C., Mei-Li, Z. ve Ji-Jiang, W., Cooperation of coordinative and $\pi \cdot \pi$ interaction in directing two-fold interpenetrated architecture of two Eu/Dy(III) 2-sulfoterephthalate complexes with 4,4-bipyridine, Zeitschrift für Anorganische und Allgemeine Chemie, 641, (3-4), 525-528, (2015).

[15] Hong-Ping, X., You-Xuan, Z., Xiao-Qiang, L., Jing-Lin, $Z$. ve Xiao-Zeng, Y., Hydrothermal synthesis, crystal structures, and luminescent properties of two lanthanide (III) complexes containing 2sulfoterephthalate, Journal of Molecular Structure, 888, 55-61, (2008).
[16] Lyszczek, R.M., Thermal investigations of cefadroxil complexes with transition metals, Journal of Thermal Analysis and Calorimetry, 78, 473, (2004).

[17] Li, X., Lu, Y., Bing, Y. ve Zha, M-Q., Synthesis, photoluminescent, and magnetic properties of two lanthanide sulfosalicylate complexes, Synthesis and Reactivity in Inorganic, Metal-Organic and Nano-Metal Chemistry, 42, 698-704, (2012).

[18] Li, X., Xie, Z., Lin, J. ve Cao, R., Lanthanide-organic frameworks constructed from multi-functional ligands: Syntheses, structures, near-infrared and visible photoluminescence properties, Journal of Solid State Chemistry, 182, 2290-2297, (2009).

[19] Feng, X., Feng, Y.Q., Chen, J., Ng, S.W., Wang, L.Y. ve Guo, J.Z., Reticular threedimensional 3d-4f frameworks constructed through substituted imidazole-dicarboxylate: syntheses, luminescence and magnetic properties study, Dalton Transactions, 44, 804-816, (2015).

[20] Lever, A.B.P., Inorganic electronic spectroscopy, Amsterdam, The Netherlands: Elsevier, (1984). 